\title{
Efficacy and safety of electroacupuncture combined with Suanzaoren decoction for insomnia following stroke: study protocol for a randomized controlled trial
}

Hui-lian Huang ${ }^{1,2+}$, Song-bai Yang ${ }^{2 \dagger}$, Zhi-gang Mei ${ }^{3,4^{*}} \mathbb{D}$, Ya-guang Huang ${ }^{5}$, Mao-hua Chen², Qun-li Mei ${ }^{2}$, Hua-ping Lei' ${ }^{2}$, Qing-xian $\mathrm{Mei}^{2}$ and Jian-hua Chen ${ }^{2}$

\begin{abstract}
Background: Insomnia is a common but frequently overlooked sleep disorder after stroke, and there are limited effective therapies for insomnia following stroke. Traditional Chinese medicine (TCM), including acupuncture and the Chinese herbal medication (CHM) Suanzaoren decoction (SZRD), has been reported as an alternative option for insomnia relief after stroke in China for thousands of years. Here, this study aims to investigate the efficacy and safety of electroacupuncture (EA) in combination with SZRD in the treatment of insomnia following stroke.

Methods: A total of 240 patients with post-stroke insomnia will be included and randomized into four groups: the EA group, SZRD group, EA \& SZRD group, and sham group. The same acupoints (GV20, GV24, HT7, and SP6) will be used in the EA group, EA \& SZRD group, and sham group, and these patients will receive the EA treatment or sham manipulation every other day for 4 consecutive weeks. SZRD treatments will be given to participants in the SZRD group and EA \& SZRD group twice a day for 4 consecutive weeks. The primary outcome measures include Pittsburgh Sleep Quality Index scores and polysomnography. Secondary outcome measures include the Insomnia Severity Index, the National Institutes of Health Stroke Scale, the Hospital Anxiety and Depression Scale, brain magnetic resonance imaging, functional magnetic resonance imaging, and nocturnal melatonin concentrations. The primary and secondary outcomes will be assessed at baseline (before treatment), during the 2nd and 4th weeks of the intervention, and at the 8th and 12th weeks of follow-up. Safety assessments will be evaluated at baseline and during the 4th week of the intervention.
\end{abstract}

Discussion: This study will contribute to assessing whether the combination of these two therapies is more beneficial for post-stroke insomnia than their independent use, and the results of this clinical trial will improve our understanding of the possible mechanisms underlying the effects of combination therapies.

\footnotetext{
* Correspondence: zhigangmei@139.com

${ }^{+}$Hui-lian Huang and Song-bai Yang contributed equally to this work.

${ }^{3}$ Key Laboratory of Hunan Province for Integrated Traditional Chinese and Western Medicine on Prevention and Treatment of Cardio-Cerebral Diseases, Hunan University of Chinese Medicine, Changsha 410208, Hunan, China

${ }^{4}$ Medical College of China Three Gorges University, Yichang 443002, Hubei,

China

Full list of author information is available at the end of the article
}

(c) The Author(s). 2021 Open Access This article is licensed under a Creative Commons Attribution 4.0 International License, which permits use, sharing, adaptation, distribution and reproduction in any medium or format, as long as you give appropriate credit to the original author(s) and the source, provide a link to the Creative Commons licence, and indicate if changes were made. The images or other third party material in this article are included in the article's Creative Commons licence, unless indicated otherwise in a credit line to the material. If material is not included in the article's Creative Commons licence and your intended use is not permitted by statutory regulation or exceeds the permitted use, you will need to obtain permission directly from the copyright holder. To view a copy of this licence, visit http://creativecommons.org/licenses/by/4.0/. The Creative Commons Public Domain Dedication waiver (http://creativecommons.org/publicdomain/zero/1.0/) applies to the data made available in this article, unless otherwise stated in a credit line to the data. 
Trial registration: Chinese Clinical Trials Register ChiCTR2000031413. Registered on March 30, 2020

Keywords: Electroacupuncture, Suanzaoren decoction, Insomnia, Stroke, Randomized controlled trial

\section{Administrative information}

\begin{tabular}{|c|c|}
\hline Title $\{1\}$ & $\begin{array}{l}\text { Efficacy and safety of } \\
\text { electroacupuncture combined with } \\
\text { Suanzaoren decoction for insomnia } \\
\text { following stroke: protocol for a } \\
\text { randomized controlled trial }\end{array}$ \\
\hline Trial registration $\{2 \mathrm{a}$ and $2 \mathrm{~b}\}$. & $\begin{array}{l}\text { Chinese Clinical Trials Register, ID: } \\
\text { ChiCTR2000031413. Registered on } \\
\text { March 30, } 2020 .\end{array}$ \\
\hline Protocol version $\{3\}$ & Date January 16, 2020 and version 2.0 \\
\hline Funding $\{4\}$ & $\begin{array}{l}\text { The study was supported by Yichang } \\
\text { Municipal Commission of Science and } \\
\text { Technology (A19-301-42) }\end{array}$ \\
\hline Author details $\{5 \mathrm{a}\}$ & $\begin{array}{l}\text { 1. School of Basic Medical Sciences, } \\
\text { Zhejiang Chinese Medical University, } \\
\text { Hangzhou, Zhejiang } 310053 \text {, China } \\
\text { 2. College of Traditional Chinese } \\
\text { Medicine, China Three Gorges } \\
\text { University \& Yichang Hospital of } \\
\text { Traditional Chinese Medicine, Yichang, } \\
\text { Hubei } 443003 \text {, China } \\
\text { 3. Key Laboratory of Hunan Province for } \\
\text { Integrated Traditional Chinese and } \\
\text { Western Medicine on Prevention and } \\
\text { Treatment of Cardio-Cerebral Diseases, } \\
\text { Hunan University of Chinese Medicine, } \\
\text { Changsha, Hunan } 410208, \text { China } \\
\text { 4. Medical College of China Three } \\
\text { Gorges University, Yichang, Hubei } \\
\text { 443002, China } \\
\text { 5. Affiliated Renhe Hospital of China } \\
\text { Three Gorges University, Yichang, Hubei } \\
\text { 443001, China }\end{array}$ \\
\hline $\begin{array}{l}\text { Name and contact information } \\
\text { for the trial sponsor }\{5 b\}\end{array}$ & $\begin{array}{l}\text { College of Traditional Chinese Medicine, } \\
\text { China Three Gorges University \& } \\
\text { Yichang Hospital of Traditional Chinese } \\
\text { Medicine, Yichang, Hubei 443003, China }\end{array}$ \\
\hline Role of sponsor $\{5 c\}$ & $\begin{array}{l}\text { The study funder has no role in the } \\
\text { study design, data collection and } \\
\text { management, and manuscript writing } \\
\text { publication }\end{array}$ \\
\hline
\end{tabular}

\section{Introduction}

\section{Background and rationale $\{6 \mathrm{a}\}$}

Stroke is the second leading cause of death and a major cause of disability worldwide. It has become one of the major contributors to the increased prevalence, incidence, and mortality of vascular disease [1]. Over 2 million people suffer a stroke in China every year [2]. Insomnia is the most common disorder of the sleepwake cycle, affecting approximately $50 \%$ of stroke survivors [3]. Insomnia is defined as repeated difficulties in initiating or maintaining sleep or early awakening accompanied by daytime functional impairment [4]. In some cases, insomnia that develops in young adulthood may be a higher risk factor for stroke than insomnia that develops later [5]. On the other hand, insomnia may directly result from cerebral ischaemia or anoxia during stroke or environmental conditions in inpatient wards or stroke-related medical conditions such as pain, depression, infections, and druginduced side effects $[6,7]$. Insomnia following a stroke can deteriorate cognitive and physical function, contributing to a worse outcome during rehabilitation [8]. Currently, the American Academy of Sleep Medicine guideline recommends cognitive behaviour therapy as a treatment for insomnia, but it is not easily or widely available [9]. Meanwhile, the side effects of hypnotic medications, such as drowsiness, fatigue, and dizziness, limit their usage in patients with post-stroke insomnia [10]. Thus, owing to the lower side effects and holistic efficacy, complementary and alternative therapies, including acupuncture and/or Chinese herbal medication $(\mathrm{CHM})$, have been incorporated into the treatment of insomnia following stroke worldwide [11].

Electroacupuncture (EA), a new form of acupuncture where a small electric current is passed between pairs of acupuncture needles, has shown positive effects on several biological parameters, such as enhancing cognitive function, learning, memory, and sleep following stroke [12]. EA beneficially regulates a healthy mental state by stimulating some special meridian points [13]. Based on theories in traditional Chinese medicine (TCM), the acupoints of the Governor meridian (Dumai in Chinese) and the Shaoyin meridian (Shaoyin Jing in Chinese) are the most common acupoints used for the treatment of stroke and its complications [14]. The distal acupoints are commonly matched with the local acupoints based on the mechanism of the disease in accordance with the theory of TCM. A systematic review of the effects of acupuncture on insomnia after stroke has shown potential benefits in the treatment of insomnia and for improving patients' sleep quality [15].

Chinese medicinal herbs and formulas have also been widely used to treat some neurological diseases, such as stroke, depression, and insomnia, in China and some Asian countries for thousands of years [16]. In recent decades, some of them have also been recommended worldwide to alleviate insomnia [17]. Suanzaoren decoction (SZRD), a well-known Chinese medicinal formula for insomnia, is a combination of five CHMs based on TCM theory and includes Suanzaoren (Ziziphi Spinosae Semen), Fuling (Poria cocos), Chuanxiong 
(Ligusticum wallichii), Zhimu (Anemarrhenae), and Gancao (Glycyrrhiza) [18]. A previous meta-analysis showed that SZRD solely or combined with other treatments could promote sleep quality and prolong sleep duration [19].

Therefore, combining EA with SZRD into a treatment method can potentially be used in the clinical therapy of post-stroke insomnia patients.

\section{Objectives \{7\}}

Although some studies have shown acupuncture combined with SZRD is more beneficial for insomnia $[20,21]$, whether EA combined with SZRD is more effective and safer for insomnia after stroke needs more clinical and experimental evidence. Therefore, we will combine EA with SZRD as a clinical treatment method for patients with post-stroke insomnia. We aim to assess whether the combination therapies are more effective and safer for insomnia after stroke than independent usage of the two treatments and to preliminarily uncover the possible mechanisms underlying the effect of the combination therapy.

\section{Trial design $\{\mathbf{8}\}$}

The study is designed as a 12-week, single-centre, randomized, single-blind, controlled trial. Study participants will be included according to the inclusion and exclusion criteria. Before starting the treatment, all eligible patients will undergo a standard neuroimaging examination (brain magnetic resonance imaging and functional magnetic resonance imaging), a baseline evaluation of sleep quality, neurological deficits, anxiety and depression, and measurement of nocturnal melatonin concentrations. Then, subjects will be equally randomized into four groups: the EA group, SZRD group, EA \& SZRD group, and sham group. Patients in the EA group and sham group will receive EA stimulation or sham EA every other day for 4 consecutive weeks for a total of 14 sessions. Patients in the SZRD group will only take SZRD granules orally twice a day for 4 consecutive weeks. Patients in the EA \& SZRD group will be administrated SZRD granules orally twice a day for 4 consecutive weeks, combined with EA stimulation every other day for 4 consecutive weeks, for a total of 14 sessions. Assessments will be carried out at baseline (before treatment), during the 2nd and 4th weeks of the intervention, and at the 8th and 12th weeks of follow-up.

The trial will be performed according to the principles of the Declaration of Helsinki (Fortaleza version, 2013), the Consolidated Standards of Reporting Trials [22], the Standards for Reporting Interventions in Clinical Trials of Acupuncture [23], and Recommendations for Intervention Trials (SPIRIT) Checklist.

\section{Methods: participants, interventions, and outcomes Study setting $\{9\}$}

A total of 240 patients with post-stroke insomnia will be recruited through advertisements on the WeChat official platform and hospital bulletin boards at the College of Traditional Chinese Medicine, China Three Gorges University \& Yichang Hospital of Traditional Chinese Medicine. Written informed consent will be provided by all patients at the time of recruitment. Patients will have an equal chance of being randomly allocated to the EA group, SZRD group, EA \& SZRD group, and sham group. Due to the limitations of the intervention methods, the therapists will not be blinded. The assessors will perform the evaluations and analysis of the outcomes at five points (before treatment, during the 2nd and 4th weeks of the intervention, and the 8th and 12th weeks of follow-up). Data management and statistics will be conducted at the Medical College of China Three Gorges University. The flow chart is shown in the SPIR IT Figure (Fig. 1).

\section{Eligibility criteria $\{10\}$ Inclusion criteria}

Participants who meet the following criteria will be included in the trial:

1. Aged $18-70$ years, either sex;

2. Fulfilling the diagnostic criteria of stroke based on the Chinese guidelines for diagnosis and treatment of acute ischemic stroke 2018 [24] or Chinese guidelines for diagnosis and treatment of acute intracerebral haemorrhage 2019 [25];

3. After acute stroke; and

4. Fulfilling the diagnostic criteria of insomnia according to the Diagnostic and Statistical Manual of Mental Disorders, fifth edition [4].

\section{Exclusion criteria}

Participants who meet the following criteria will be excluded from the trial:

1. Had insomnia before stroke;

2. Insomnia by poor sleep conditions [26];

3. Have diagnoses of other sleep disorders, such as narcolepsy, obstructive sleep apnoea syndrome, or restless legs syndrome;

4. Have neurological or psychiatric diseases;

5. Have severe diabetes; hypertension; severe liver, kidney, or thyroid dysfunction; and severe cardiac insufficiency;

6. Are unable to read, understand, and complete the forms or use the rating scales; 


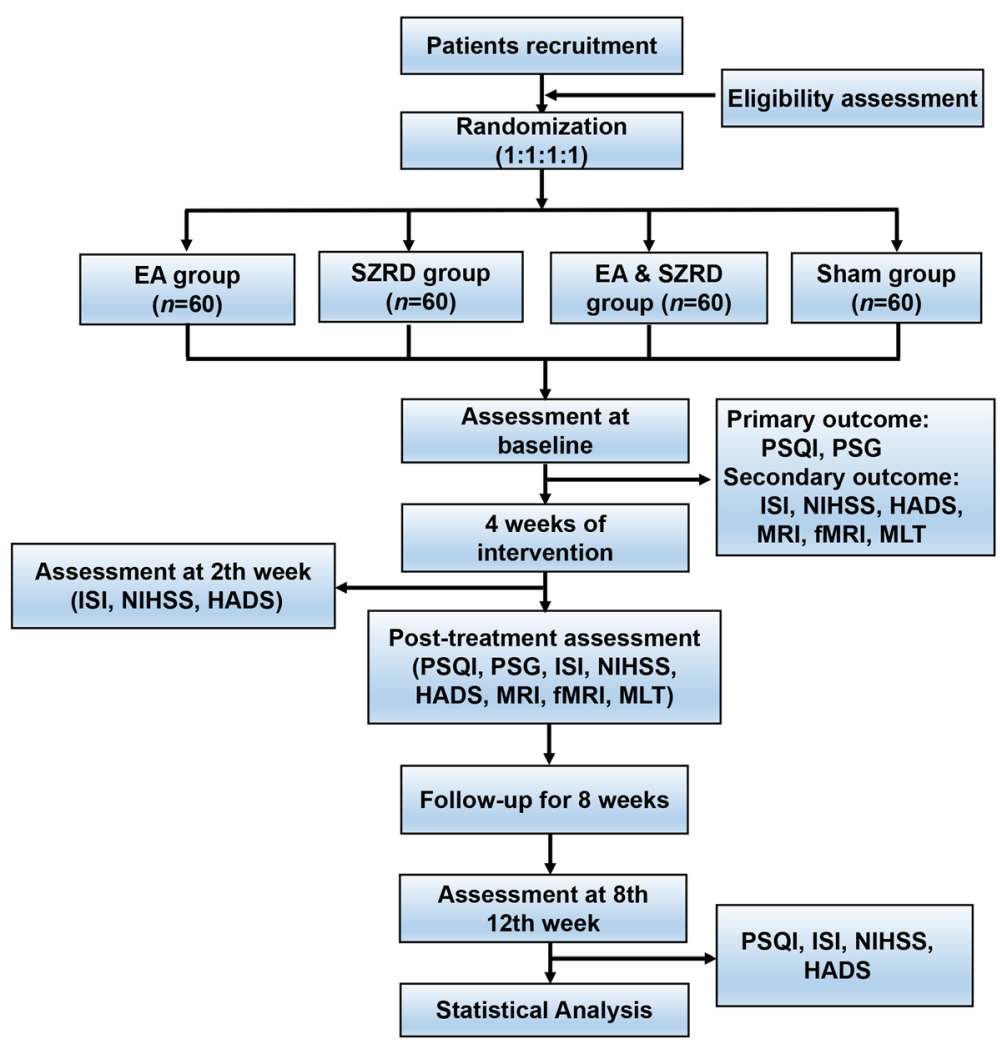

Fig. 1 Flow chart. EA, electroacupuncture; SZRD, Suanzaoren decoction; PSQI, Pittsburgh Sleep Quality Index; PSG, polysomnography; ISI, Insomnia Severity Index; NIHSS, National Institutes of Health Stroke Scale; HADS, Hospital Anxiety and Depression Scale; MRI, brain magnetic resonance imaging; fMRl, functional magnetic resonance imaging; MLT, nocturnal concentration of melatonin

7. Have implants that could interfere with EA or a history of hypersensitivity to EA;

8. Had received TCM in the previous 1 month;

9. Had participated in other trials;

10. Are taking sleep-affecting diet or medication; or

11. Are pregnant or preparing for pregnancy.

\section{Who will take informed consent? $\{26 a\}$}

The principal investigator at Yichang Hospital of Traditional Chinese Medicine will conduct the informed consent process, who will screen the potential participants in accordance with the inclusion and exclusion criteria. Written informed consent will be provided by eligible patients at the time of recruitment.

\section{Additional consent provisions for collection and use of participant data and biological specimens $\{26 \mathrm{~b}\}$}

During the intervention period, patients will have the right to withdraw for whatever reason and at whatever time. Therefore, on the consent form, participants are asked if they agree to the use of their data when they choose to withdraw from the trial. Participants will also be asked for permission for the research team to share relevant data with people from the Medical College of
China Three Gorges University or Yichang Hospital of Traditional Chinese Medicine. This trial does not involve collecting biological specimens that will be stored.

\section{Interventions}

Explanation for the choice of comparators $\{6 b\}$

In this study, we will assess whether EA combined with SZRD is more beneficial for post-stroke insomnia than independent usage of the two treatments. Therefore, EA, SZRD, and sham EA were chosen as comparators in this trial.

\section{Intervention description $\{11 \mathrm{a}\}$}

All manipulators with acupuncturist qualification certificates have independently performed clinical treatments for more than 2 years. The acupuncturists will engage in the clinical training before the intervention to ensure experience with the standard real and sham acupuncture operations and will not be replaced during the trial.

Disposable acupuncture needles $(0.35 \times 25 \mathrm{~mm}$ or 0.35 $\times 40 \mathrm{~mm}$, JiaJian Medical Instrument Co., Ltd, Wuxi, China), sham acupuncture needles with blunt tips $(0.30$ $\times 25 \mathrm{~mm}$ ), G6805-2A EA devices (Huayi Medical 
Instrument Co., Ltd, Shanghai, China), and SZRD granules (Tcmages Pharmaceutical Co., Ltd, Beijing, China) will be used in this trial. The acupuncturists will use 0.35 $\times 25 \mathrm{~mm}$ needles for acupoints GV20, GV24, and HT7 and $0.35 \times 40 \mathrm{~mm}$ needles for acupoint SP6. The depth of needle insertion will be $10-25 \mathrm{~mm}$ for each acupoint. Participants will receive EA or sham EA treatment every other day for 4 weeks for a total of 14 sessions, and participants receiving SZRD treatment will take $3.4 \mathrm{~g}$ SZRD granules orally twice a day for 4 weeks. After the baseline assessment, it will take 12 weeks for a patient to complete the trial, including 4 weeks of treatment and 8 weeks of follow-up (the intervention process chart is shown in Fig. 2B). Before the manual operation, patients will be asked to wear eye masks and lie supine. Each session will last for $30 \mathrm{~min}$. The temperature in the isolated treatment room will be $25^{\circ} \mathrm{C}$ (the interventions for all groups are shown in Table 1$)$.

\section{EA group}

Subjects in this group will receive EA treatment. The following acupoints were selected: local acupoints: Baihui (GV20) and Shenting (GV24); distal acupoints: bilateral Shenmen (HT7) and bilateral Sanyinjiao (SP6). After skin disinfection, sterile adhesive pads will first be fixed on the acupoints, and the acupuncture needles will be inserted through the adhesive pads into the skin. Needle insertion will follow an angle of $30^{\circ}$ along the anterior-posterior midline for GV20 and GV24. Perpendicular punctures will be used for HT7 and SP6. Acupuncturists will manipulate the needle until patients experience the de qi sensation (manifesting as soreness,

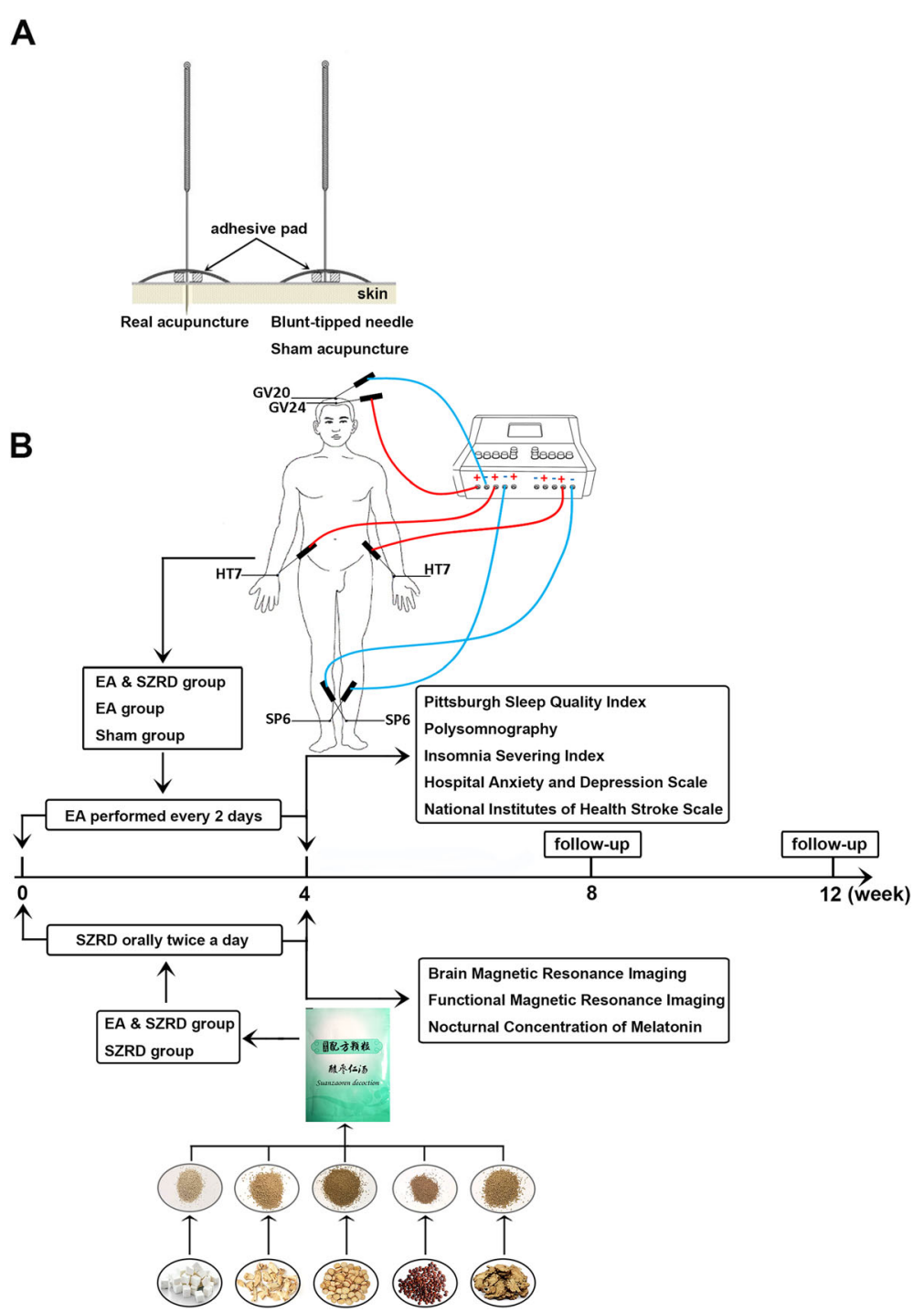

Fig. 2 The manipulation of the real and sham acupuncture device and intervention process chart. A The manipulation of the real and sham acupuncture. $\mathbf{B}$ The intervention process chart 
Table 1 Intervention parameters of each group

\begin{tabular}{|c|c|c|c|c|}
\hline \multirow[t]{2}{*}{ Groups } & \multicolumn{4}{|l|}{ Interventions } \\
\hline & Acupoints & EA parameters & Chinese herbs & Treatment sessions \\
\hline $\begin{array}{l}\text { EA \& } \\
\text { SZRD } \\
\text { group }\end{array}$ & $\begin{array}{l}\text { Bilateral HT7, bilateral SP6, } \\
\text { GV20, GV24 }\end{array}$ & $\begin{array}{l}\text { Continuous wave stimulation; } \\
\text { frequency }=10 \mathrm{~Hz} \text {; current ranges } \\
\text { from } 1 \text { to } 5 \mathrm{~mA}\end{array}$ & $\begin{array}{l}\text { Suanzaoren, Zhimu, } \\
\text { Fuling, Chuanxiong, } \\
\text { Gancao }\end{array}$ & $\begin{array}{l}4 \text { weeks, EA performed every other day, total } \\
14 \text { sessions; SZRD granules orally taken twice } \\
\text { a day }\end{array}$ \\
\hline $\begin{array}{l}\text { EA } \\
\text { group }\end{array}$ & $\begin{array}{l}\text { Bilateral HT7, bilateral SP6, } \\
\text { GV20, GV24 }\end{array}$ & $\begin{array}{l}\text { Continuous wave stimulation; } \\
\text { frequency }=10 \mathrm{~Hz} \text {; current ranges } \\
\text { from } 1 \text { to } 5 \mathrm{~mA}\end{array}$ & No herbs & $\begin{array}{l}4 \text { weeks, EA performed every other day, total } \\
14 \text { sessions }\end{array}$ \\
\hline $\begin{array}{l}\text { SZRD } \\
\text { group }\end{array}$ & No acupoints & No parameters & $\begin{array}{l}\text { Suanzaoren, Zhimu, } \\
\text { Fuling, Chuanxiong, } \\
\text { Gancao }\end{array}$ & $\begin{array}{l}4 \text { weeks, SZRD granules orally taken twice a } \\
\text { day }\end{array}$ \\
\hline $\begin{array}{l}\text { Sham } \\
\text { group }\end{array}$ & $\begin{array}{l}\text { Bilateral HT7, bilateral SP6, } \\
\text { GV20, GV24 (no need } \\
\text { inserting) }\end{array}$ & Frequency $=0 \mathrm{~Hz}$; current $=0 \mathrm{~mA}$ & No herbs & $\begin{array}{l}4 \text { weeks, sham EA performed every other day, } \\
\text { total } 14 \text { sessions }\end{array}$ \\
\hline
\end{tabular}

fullness, heaviness, and numbness when lifting, thrusting, or rotating the needle). There are three sets of electrodes connected. One set of two electrodes of the EA device are connected at GV20 and GV24. Another two sets of electrodes are connected at the ipsilateral HT7 and SP6. The electroacupuncture intervention will apply $10-\mathrm{Hz}$ continuous waves, and the current will range from 1 to $5 \mathrm{~mA}$. The intensity will be adjusted based on the tolerance of each patient. Each session will last $30 \mathrm{~min}$ and be performed every other day for 4 successive weeks, for a total of 14 sessions.

\section{SZRD group}

Subjects in this group will receive SZRD treatment. SZRD consists of 5 herbs, including Suanzaoren, Fuling, Zhimu, Chuanxiong, and Gancao, in the following concentrations: $41 \%, 20 \%, 20 \%, 13 \%$, and $6 \%$, respectively. Patients will be administered SZRD granules in one bag ( $3.4 \mathrm{~g}$ per bag) dissolved in boiling water two times daily for 4 successive weeks.

\section{EA \& SZRD group}

Subjects in this group will receive the EA and SZRD treatments. The procedures for the EA and SZRD treatments will be the same as those for the EA group and SZRD group. EA will last $30 \mathrm{~min}$ in each session, which will be performed every other day for 4 successive weeks, for a total of 14 sessions. SZRD treatment will be given two times daily for 4 successive weeks.

\section{Sham group}

Subjects in this group will receive sham EA treatment with sham acupuncture needles. The acupoints selected, manipulation procedures, electrode placements, and other treatment settings will be the same as in the EA group, while the effects of de qi will not be produced through skin penetration or needle manipulation. Furthermore, all parameters of the electrical stimulation will be set to 0 . After retaining the needles for $30 \mathrm{~min}$, acupuncturists will remove all needles using dry cotton balls and press the acupoints to provide patients with the sensation of a 'real' needle being withdrawn from the skin. Each session will last $30 \mathrm{~min}$ and will be performed every other day for 4 successive weeks.

\section{Criteria for discontinuing or modifying allocated interventions $\{11 \mathrm{~b}\}$}

If participants feel that their diseases are not improving, they will be free to withdraw from the clinical study and choose other therapies. Participants who meet the following criteria could be discontinued from receiving the interventions:

1. They develop a serious disease such as heart disease, pneumonia, or stroke during the trial;

2. They receive other treatments for insomnia such as other medicine, moxibustion, or massage, during the study;

3. They experience serious side effects;

4. They refuse to continue to participate in the RCT;

5. They are lost to follow-up; or

6. They do not comply with the study protocol.

\section{Strategies to improve adherence to interventions $\{11 c\}$}

Frequent follow-up telephone calls and messages on the WeChat platform will be important aspects for improving adherence to interventions.

\section{Relevant concomitant care permitted or prohibited during the trial $\{11 \mathrm{~d}\}$}

Given the history of stroke, all participants will continue to take basic Western medications for the secondary prevention of stroke during the study. The name, dose, and usage of the medications will be recorded.

\section{Provisions for post-trial care $\{30\}$}

If the condition of a subject worsens after the trial or is accompanied by severe complications or serious adverse 
reactions, the specialist will take medical measures to deal with the harm based on the subject's condition. The treatments will be free.

\section{Outcomes $\{12\}$}

\section{Primary outcomes}

The following primary outcomes will help us to assess whether the combination of EA with SZRD is more effective in insomnia in post-stroke patients.

Pittsburgh Sleep Quality Index The PSQI is a selfrating questionnaire scale with 19 self-rated items and five other-rated items measuring sleep quality and disturbances over a period of 1 month [27]. The self-rated items include the following seven subscales: sleep quality, sleep latency, sleep duration, habitual sleep efficacy, sleep disturbances, use of sleeping medication, and daytime dysfunction. Each subscale will be answered by patients on a 0 to 3 response category scale, and the total score of the PSQI can range from 0 to 21 points. A lower score indicates a better quality of sleep. The assessment will be performed at baseline (before treatment) and during the 4th week of the intervention, as well as at the 8th and 12th weeks of follow-up.

Polysomnography PSG (Curative Medical Inc, Santa Clara, CA, USA) is used to objectively estimate sleep [28]. It records quantifiable data during sleep, such as total sleep time, sleep onset latency, waking after sleep onset, rapid eye movement, and non-rapid eye movement sleep. In this study, all patients will be assessed for two nights (one night at baseline and one night at 4 weeks post-treatment) in a specialized sleep laboratory. During the assessment process, the sleep time of participants will be based on their habit. Standard equipment will record sleep conditions from 22:00 to 7:00 for each assessment. All PSG data will be further analysed by Acumen 7 EV 2.11 software.

\section{Secondary outcomes}

The following secondary outcomes will help us to determine whether participants with post-stroke insomnia in the EA \& SZRD group show greater improvements in sleep quality and other related symptoms than the other three groups after the therapies.

Insomnia Severity Index The ISI is a self-report questionnaire designed to assess the degree of insomnia and the associated functional impairments [29]. The total score on the ISI can range from 0 to 28 points. A score of 0 to 7 indicates no clinically significant insomnia. A score of 8 to 14 is regarded as sub-threshold insomnia. A score of 15 to 21 is regarded as clinical insomnia of moderate severity. More than 22 points is regarded as severe clinical insomnia. The assessment will be performed at baseline (before treatment) and during the 2nd and 4th weeks of the intervention, as well as at the 8th and 12th weeks of follow-up.

Hospital Anxiety and Depression Scale The HADS is a brief assessment used to measure current anxiety and depressive symptoms in a non-psychiatric hospital patient [30]. The HADS comprises two independent sevenitem subscales for anxiety and depression. More than 11 points on either subscale indicate the probable presence of the mood disorder. The assessment will be performed at baseline (before treatment) and during the 2nd and 4th weeks of the intervention, as well as at the 8th and 12th weeks of follow-up.

National Institutes of Health Stroke Scale The NIHSS is a stroke-specific quantitative scale used to assess neurological deficits [31]. Each participant will be assessed by a trained, certified investigator at baseline (before treatment) and during the 2nd and 4th weeks of the intervention, as well as at the 8th and 12th weeks of follow-up. A higher score indicates a more severe neurological deficit.

Brain magnetic resonance imaging (MRI) MRI is a non-invasive imaging method to detect features of brain tissue [32]. In particular, MRI is a well-suited technique to visualize and analyse the anatomical properties and abnormalities in the brain. Each participant will be assessed at baseline (before treatment) and during the 4 th week of the intervention.

Functional magnetic resonance imaging (fMRI) fMRI is another non-invasive technique that is used to track metabolic activity in the brain. Abnormal fMRI might serve as a potential neuromarker for insomnia [33]. Each participant will be assessed at baseline (before treatment) and during the 4th week of the intervention.

Nocturnal concentration of melatonin (MLT) Melatonin coordinates circadian rhythms and regulates sleep function [34]. Blood samples of patients will be collected at 3:00 a.m. in a dimly lit environment to diminish the impact of light on the secretion of melatonin. Each participant will be assessed at baseline (before treatment) and during the 4th week of the intervention.

\section{Safety assessments}

Safety will be assessed by renal function tests, liver function tests, routine blood tests, routine urine tests, routine stool tests, and electrocardiograms. These 
indicators will be detected at baseline (before treatment) and during the 4 th week of the intervention.

\section{Participant timeline $\{13\}$}

Details of the case distribution, specific measurements, and time points of data capture can be found in the SPIR IT Figure (Fig. 3).

\section{Sample size $\{14\}$}

PSQI scores will be presented as the primary outcome. The sample size was calculated based on changes in PSQI scores. Based on a previous study [35], the global PSQI score after 4 weeks of treatment was $9.21 \pm 4.05$ in the intervention group and $13.18 \pm 4.32$ in the control group. We used the following formula to calculate the sample size.

$$
n_{1}=n_{2}=\frac{\left(Z_{\alpha}+Z_{\beta}\right)^{2} * 2 \sigma^{2}}{\delta^{2}}
$$

On the basis of 0.9 power to detect a significant difference ( $\alpha=0.05$, two-sided), 25 participants are required for each group. Allowing for a $20 \%$ drop-out rate, a sample size of 30 for each group is needed. To improve the RCT quality and reduce the bias, we decided to recruit a sample size of 60 for each group. As a result, we plan to recruit a total of 240 patients for the whole trial.

\begin{tabular}{|c|c|c|c|c|c|}
\hline \multirow[b]{2}{*}{ TIMEPOINT (weeks) } & \multirow[t]{2}{*}{ Baseline } & \multicolumn{2}{|c|}{ Treatment period } & \multicolumn{2}{|c|}{ Follow-up period } \\
\hline & & 2 & 4 & 8 & 12 \\
\hline \multicolumn{6}{|l|}{ ENROLMENT: } \\
\hline \multirow{4}{*}{$\begin{array}{l}\text { Eligibility screen } \\
\text { Informed consent } \\
\text { Medical history } \\
\text { Randomization }\end{array}$} & $x$ & & & & \\
\hline & $x$ & & & & \\
\hline & $x$ & & & & \\
\hline & $x$ & & & & \\
\hline \multicolumn{6}{|l|}{ INTERVENTIONS: } \\
\hline \multicolumn{6}{|l|}{ EA group } \\
\hline \multicolumn{6}{|l|}{ SZRD group } \\
\hline \multicolumn{6}{|l|}{ EA \& SZRD group } \\
\hline \multicolumn{6}{|l|}{ Sham group } \\
\hline \multicolumn{6}{|l|}{ ASSESSMENTS: } \\
\hline \multicolumn{6}{|l|}{ Primary outcome: } \\
\hline PSQI & $x$ & & $x$ & $x$ & $x$ \\
\hline PSG & $x$ & & $x$ & & \\
\hline \multicolumn{6}{|l|}{ Secondary outcome: } \\
\hline ISI & $x$ & $x$ & $x$ & $x$ & $x$ \\
\hline NIHSS & $x$ & $x$ & $x$ & $x$ & $x$ \\
\hline HADS & $x$ & $x$ & $x$ & $x$ & $x$ \\
\hline MRI & $x$ & & $x$ & & \\
\hline fMRI & $x$ & & $x$ & & \\
\hline MLT & $x$ & & $x$ & & \\
\hline \multicolumn{6}{|l|}{ Safe outcome: } \\
\hline \multirow{3}{*}{$\begin{array}{l}\text { Blood routine test } \\
\text { Urine routine test } \\
\text { Stool routine test }\end{array}$} & $x$ & & $x$ & & \\
\hline & $x$ & & $x$ & & \\
\hline & $x$ & & $x$ & & \\
\hline \multirow{4}{*}{$\begin{array}{l}\text { Liver function tests } \\
\text { Kidney tests } \\
\text { Electrocardiogram exam } \\
\text { Adverse events }\end{array}$} & $x$ & & $x$ & & \\
\hline & $x$ & & $x$ & & \\
\hline & $x$ & & $x$ & & \\
\hline & & $x$ & $x$ & $x$ & $x$ \\
\hline
\end{tabular}

Fig. 3 Details of measurements, and time points of data collection. EA, electroacupuncture; SZRD, Suanzaoren decoction; PSQI, Pittsburgh Sleep Quality Index; PSG, polysomnography; ISI, Insomnia Severity Index; NIHSS, National Institutes of Health Stroke Scale; HADS, Hospital Anxiety and Depression Scale; MRI, brain magnetic resonance imaging; fMRI, functional magnetic resonance imaging; MLT, nocturnal concentration of melatonin 


\section{Recruitment $\{15\}$}

Patients with post-stroke insomnia will be recruited through advertisements on the WeChat official platform and hospital bulletin boards. If patients are interested in this study, the research coordinator will obtain their contact details. Then, the principal investigator will make an appointment with each successive patient. In an individual room, the potential participant will be told in detail of the purpose and content of the study as well as the benefits and risks of participating by the principal investigator, who will screen them based on the inclusion and exclusion criteria and obtain informed consent from the eligible patients.

\section{Assignment of interventions: allocation Sequence generation $\{16 \mathrm{a}\}$}

The investigator at Yichang Hospital of Traditional Chinese Medicine will generate random numbers 001240 by SPSS 20.0 software (SPSS Inc., Chicago, IL, USA) and places random numbers in a sequenced, sealed, opaque envelope.

\section{Concealment mechanism $\{16 b\}$}

The random numbers in the sequenced sealed opaque envelopes will have been equally allocated to the four groups $(0,1,2$, or 3 group). The principal investigator, who will enrol the eligible patients, will open the envelopes based on patients' registration order numbers and then assign the patients to groups $0,1,2$, or 3 in accordance with the group number in the envelope. The random numbers will be placed in opaque sealed envelopes to prevent selection bias. The researchers, data collection staff, and data analysts will be blinded in the course of the random allocation sequence to avoid detection bias.

\section{Implementation $\{16 \mathrm{c}\}$}

The designated investigator will generate the allocation sequence, and another investigator designated by the project leader will enrol and assign participants.

\section{Assignment of interventions: blinding \\ Who will be blinded $\{17 a\}$}

We designed a single-blind trial in which the participants of the EA group, the participants of the sham EA group, data collection staff, and data analysts will be blinded during the course of the research. Because of the particular treatment manipulations, blinding the acupuncturists, the participants of the EA \& SZRD group, and the SZRD group is impossible. During the trial process, acupuncturists and data collection staff will be forbidden to exchange information with each other and communicate with the study patients. We will conduct each treatment in a closed unit with patients wearing eye masks. To maximize blinding of participants, a sham device will be utilized [36, 37] (shown in Fig. 2A).

\section{Procedure for unblinding if needed $\{17 \mathrm{~b}\}$}

Researchers will be permitted to be unblinded in some specific situations, such as participants experiencing serious adverse events or disease progression, and where prompt treatment and appropriate management are needed. The procedure for unblinding will be as follows: (1) prior to unblinding, the researcher will need to inform the trial steering committee. (2) The researcher should unblind the intervention information of the patient based on the emergency envelope. (3) The researcher will need to fill out the report form of unblinding and note it on the case report form (CRF).

\section{Data collection and management}

Plans for assessment and collection of outcomes $\{18 \mathrm{a}\}$

All data will be documented in detail in the CRF after face-to-face visits. The same researcher will be responsible for the examination of each patient at each assessment. Baseline measurements, medical history, demographics, and previous medications will be collected at the first visit. The primary and secondary outcomes will be assessed at baseline (before treatment) and during the 2nd and 4th weeks of the intervention, as well as at the 8th and 12th weeks of follow-up. Safety assessments will be evaluated at baseline and during the 4th week of the intervention.

\section{Plans to promote participant retention and complete follow-up \{18b\}}

Follow-up telephone calls and messages on the WeChat public platform will be conducted to promote participant retention.

\section{Data management $\{19\}$}

Epidata 3.1 (EpiData Association, Denmark), which is a statistical package, will be used for data entry. Two trained data entry staff members will recheck the CRF and then independently perform data entry. The administrators at Medical College of China Three Gorges University will independently check and judge the input data and confirm whether all CRFs are being completed in a timely manner to ensure that the withdrawal of participants and all adverse events are recorded in CRFs.

\section{Confidentiality $\{27\}$}

Patient names in the dataset will be identified with Pinyin initials and numbers. CRFs will be stored in a securely locked location. Epidata 3.1 will be locked and analysed by an independent statistician under the supervision of the administrators. The data and relevant 
records from this trial will be input on the ResMan Research Manager website at http://www.medresman. org.cn/login.aspx.

Plans for collection, laboratory evaluation, and storage of biological specimens for genetic or molecular analysis in this trial/future use $\{33\}$

No biological specimens will be collected in this trial.

\section{Statistical methods}

Statistical methods for primary and secondary outcomes $\{20 a\}$

Based on a previous study, the minimal clinically important difference is approximately $1.14-1.75$ points on the PSQI score following treatments [38]. We will assume a treatment response with a score reduction on the PSQI of 2 or more points. Descriptive statistics will be used to analyse baseline characteristics for each group. Repeated measures analysis will be performed to make comparisons between the treatment groups and the control group (EA \& SZRD versus sham EA, EA versus sham $E A$, and $S Z R D$ versus sham $E A$ ) at different time points (2nd, 4th, 8th, and 12th weeks). If a significant difference is detected, the next step will be to make comparisons among the three treatment groups in effectiveness. The Bonferroni correction method will be used for multiple comparisons. The Kruskal-Wallis test will be used for the analysis of data with a skewed distribution. Analysis of variance will be used for numerical variables, and the chi-squared test will be used for categorical variables. SPSS 20.0 software (SPSS Inc., Chicago, IL, USA) will be used to analyse the data.

\section{Interim analyses $\{21 \mathrm{~b}\}$}

Although an interim analysis will not be conducted, the trial should be terminated if there is clear evidence that serious adverse events (SAEs) are associated with the intervention.

\section{Methods for additional analyses (e.g. subgroup analyses) \{20b\}}

Multivariate logistic regression will be used to conduct exploratory analyses. Based on insomnia duration, insomnia can be categorized into acute insomnia (lasting up to three months) and chronic insomnia (lasting at least 3 months) [39]. A subgroup analysis will be conducted based on the duration of insomnia after stroke.

\section{Methods in analysis to handle protocol non-adherence} and any statistical methods to handle missing data $\{20 \mathrm{c}\}$ The evaluation of the outcomes will be based on an intention-to-treat analysis (ITT). Before statistical analysis of the data, the missing data mechanism will be examined, and the missing data pattern will be investigated. Multiple imputation (MI) is a principled missing data method that provides valid statistical inferences under missing at random conditions [40]. Considering $20 \%$ missing data, at least five imputations will be necessary [41]. We will select the preferred imputation method based on the missing data pattern [41].

Plans to give access to the full protocol, participant-level data, and statistical code $\{31 \mathrm{c}\}$

The datasets generated and analysed during the study will be available from the corresponding author on reasonable request.

\section{Oversight and monitoring}

Composition of the coordinating centre and trial steering committee $\{5 \mathrm{~d}\}$

The coordinating group is composed of the research coordinator and nurses. Nurses are responsible for communicating with patients who are interested in the recruitment advertisement and performing the phone call consultation and WeChat visit of the participants. The research coordinator will help the principal investigator obtain the contact details of potential participants and communicate with patient advisers who provide their experiences as patients with post-stroke insomnia to the design and details of this study. The steering committee (SC) is composed of the subject leader and the project manager. The $\mathrm{SC}$ will be responsible for the surveillance of the quality control and the progress of the study. The SC will appoint inspectors as a monitor group to oversee this study in accordance with the Good Clinical Practice guidelines. The monitor group will conduct daily supervision activities and then will write the inspection report, which will mainly include the quality of the CRF form, researchers' compliance with the proto$\mathrm{col}$, and recruitment processing of patients to the steering committee every week. The SC will have the authority to allow modification of the plan.

\section{Composition of the data monitoring committee, its role, and reporting structure $\{21 \mathrm{a}\}$}

The administrators at the Medical College of China Three Gorges University will be responsible for monitoring data management as an independent third party. The administrators will write the data monitoring report, which will mainly include the accuracy of entries and the data entry staff's knowledge of various standards, to the SC every week.

\section{Adverse event reporting and harms $\{22\}$}

When adverse events (AEs) occur, it will be necessary to record the name of the $\mathrm{AE}$, the beginning and end times of the $\mathrm{AE}$, the outcomes of the $\mathrm{AE}$, the severity of the 
$\mathrm{AE}$, the treatments related to the $\mathrm{AE}$, causality, and the application of combined drugs. The researchers will analyse causality based on the following five criteria: (1) Is there a reasonable time sequence between the intervention and $\mathrm{AE}$ ? (2) Is the $\mathrm{AE}$ consistent with the type of AEs known to be associated with the intervention? (3) Did the AE disappear or decrease after discontinuation of the intervention? (4) Does the same $\mathrm{AE}$ appear when the patient receives the same intervention? (5) Can the $\mathrm{AE}$ be explained by a combination of medications or by the patient's disease progression?

AEs will include nausea, tiredness, dizziness, syncope, palpitations, hepatic dysfunction, and kidney dysfunction. SAE harms include death, life-threatening, hospital treatments, and permanent disability.

\section{Frequency and plans for auditing trial conduct $\{23\}$}

Our trial will be audited every 6 months by qualified clinical trial experts, and the review process will be independent of researchers and sponsors.

\section{Plans for communicating important protocol amendments to relevant parties (e.g. trial participants, ethical committees) $\{25\}$}

If there is any change to eligibility criteria, outcomes, or analyses, a new version of the protocol will be submitted to the Medical Ethics Committee of College of Traditional Chinese Medicine, China Three Gorges University \& Yichang Hospital of Traditional Chinese Medicine, for approval.

\section{Dissemination plans $\{31 \mathrm{a}\}$}

The results of the study will be presented in the form of publications or conference reports.

\section{Discussion}

A stroke occurs when a blood vessel that carries oxygen and nutrients to the brain is either blocked by a clot or bursts (or ruptures), which frequently leads to long-term deficits in physical and cognitive functions in patients [42]. Insomnia is one of the most common complications in stroke patients [43]. Although the exact pathophysiology between insomnia and stroke remains complicated, a basic consensus has been reached that the pathophysiological mechanisms linking insomnia with worse stroke outcomes may be relevant to sympathetic overactivation, hypoxaemia, oxidative stress, and inflammatory reactions [44]. Some studies have provided evidence that insomnia patients show inferior motor function and other worse stroke recovery, and it is believed that healthy sleep plays an important role in the restoration and compensation of neurological functions associated with stroke [45-47]. Ameliorating insomnia during any phase of stroke is positive for short- and longterm recovery [47].

SZRD, one of the most widely used CHM formulas, has been utilized for insomnia for thousands of years in China [19]. A network analysis revealed that the five herbs in SZRD may play different roles in a coordinated manner to exert a range of sedative and hypnotic actions via GABAergic and serotonergic systems [48]. GABAergic activity not only improves sleep but also promotes sleep-related learning and memory processes, which are potentially relevant to neurorehabilitation after stroke [49]. In addition, it has been found that SZRD is beneficial for balancing the nervous system, regulating blood sugar and blood pressure, normalizing the hormone system, and improving the inflammatory response [50].

EA has been used in the treatment of stroke for decades [51]. Recently, it has also been widely utilized as a clinical treatment for insomnia [52]. An evidence-based review showed that EA significantly alleviates pathological damage and promotes post-stroke functional recovery via neurogenesis and astrogliosis [53]. Accumulating data have documented that EA may effectively work by a variety of neurotransmitters, such as adenosine, GABA, galanin, and glutamate, which are related to improving sleep quality [54]. Some clinical RCTs have indicated that EA or EA combined with other therapies is effective for the treatment of insomnia $[55,56]$.

Therefore, we propose to conduct a clinical RCT on EA plus SZRD for the treatment of post-stroke insomnia. We designed a randomized, four-group, and singleblinded clinical trial with a 4-week treatment period and a 12-week follow-up period. Based on TCM theory and previous studies, Shenmen (HT7), Sanyinjiao (SP6), Baihui (GV20), and Shenting (GV24) are the most frequently selected acupoints for the treatment of poststroke insomnia, and local acupoint stimulation can improve the curative effect when combined with distal acupoints $[57,58]$. We also emphasize the experience of the "de $q i$ " sensation during the intervention. There have been differences in efficacy between low-frequency and high-frequency electrical stimulation in EA therapy [59, 60]. Accumulating data have shown that low-frequency EA is effective for complications related to stroke, such as insomnia, epilepsy, and pseudobulbar palsy [61-63]. We selected a $10-\mathrm{Hz}$ frequency of EA to treat insomnia after stroke in this trial. Preliminary analyses have indicated that different types of sham acupuncture may have different effect sizes, and a meta-analysis showed that sham devices may have minimum non-specific effects $[64,65]$. We chose to apply a sham device in the sham group to ensure the precision of the results. 
Non-invasive neuroimaging technology is the most commonly recommended diagnostic tool in stroke [66]. Technologies such as fMRI open a window for the study of insomnia after stroke. Some researchers have found that patients with primary insomnia have abnormal brain metabolism and connectivity related to particular brain regions, such as the prefrontal cortex, insular cortex, amygdala, precuneus, and caudate $[67,68]$. Thus, we can utilize fMRI to assess aspects of treatment-induced changes in resting-state brain metabolism and elucidate the possible underlying mechanisms of these therapies in this protocol.

Melatonin is a hormone secreted by the pineal body, with peak secretion at night [69]. Some researchers have found that abnormal secretion of melatonin is potentially related to insomnia in stroke patients [70, 71]. A preliminary report revealed that acupuncture increased nocturnal melatonin secretion and reduced insomnia and anxiety [72]. We will detect the nocturnal melatonin concentrations in patients with post-stroke insomnia to investigate whether EA plus SZRD can also play a beneficial role by increasing nocturnal melatonin secretion and reducing insomnia.

We hope to elucidate the possible underlying mechanisms of this combination therapy by using MRI and melatonin detection. However, our study has some limitations. One limitation is the use of self-rating scales, which might affect the evaluation of the severity of the insomnia disorder. However, we will attempt to decrease the subjective factors by PSG. Another limitation is that the trial will be performed in a single-blind manner, and it will be conducted in only one medical centre. These limitations may weaken the generalizability of the study. A large sample, multicentre, study using objective measures to assess the efficacy of EA plus SZRD treatment should be conducted in a future study.

\section{Trial status}

Recruitment began in April 2020 and the approximate date when recruitment will be completed is January 2022. Protocol version 2.0 was approved on January 16, 2020.

\section{Abbreviations \\ EA: Electroacupuncture; SZRD: Suanzaoren decoction; TCM: Traditional Chinese medicine; CHM: Chinese herb medication; PSG: Polysomnography; PSQI: Pittsburgh Sleep Quality Index; ISI: Insomnia Severity Index; HADS: Hospital Anxiety and Depression Scale; NIHSS: National Institutes of Health Stroke Scale; MRI: Brain magnetic resonance imaging; fMRI: The functional magnetic resonance imaging; MLT: Nocturnal concentration of melatonin; RCT: Randomized controlled trial; CRF: Case report form}

\section{Acknowledgements}

The authors would like to thank Xihe Huang as a patient representative, for providing his experience as a patient with post-stroke insomnia to the design and details of this trial, and other patient advisers.
Authors' contributions $\{31 \mathrm{~b}\}$

ZGM conceived and designed the study. SBY participated in designing the study and is in charge of the treatment of patients in the EA plus SZRD group. HLH drafted the manuscript and was responsible for obtaining approval from the Medical Ethics Committee of College of Traditional Chinese Medicine, China Three Gorges University \& Yichang Hospital of Traditional Chinese Medicine, and registering the trial with the Chinese Clinical Trial Registry. YGH contributed to drafting the manuscript and prepared the figures. MHC is responsible for the treatment of patients in the SZRD group. HPL is assigned the task of treating patients in the EA group, and JHC treats patients in the sham group. QXM is responsible for central randomization and made amendments to the manuscript. QLM takes charge of recruitment, collects data, and provides pictures. All authors read and approved the final version of the manuscript.

\section{Funding $\{4\}$}

The study was supported by the Yichang Municipal Commission of Science and Technology (A19-301-42). The study funder has no role in the study design, data collection and management, and manuscript writing publication.

Availability of data and materials $\{29\}$

Any data required to support the protocol can be supplied on request.

\section{Declarations}

Ethics approval and consent to participate $\{24\}$

The protocol has been approved by the Medical Ethics Committee of College of Traditional Chinese Medicine, China Three Gorges University \& Yichang Hospital of Traditional Chinese Medicine: the approval number is 20200302. Written consent is obtained from all participants by the researcher.

\section{Consent for publication $\{32\}$}

No personal information is provided in this manuscript.

\section{Competing interests $\{28\}$}

The authors declare that they have no competing interests.

\section{Author details}

${ }^{1}$ School of Basic Medical Sciences, Zhejiang Chinese Medical University, Zhejiang 310053, Hangzhou, China. ${ }^{2}$ College of Traditional Chinese Medicine, China Three Gorges University \& Yichang Hospital of Traditional Chinese Medicine, Yichang 443003, Hubei, China. ${ }^{3}$ Key Laboratory of Hunan Province for Integrated Traditional Chinese and Western Medicine on Prevention and Treatment of Cardio-Cerebral Diseases, Hunan University of Chinese Medicine, Changsha 410208, Hunan, China. ${ }^{4}$ Medical College of China Three Gorges University, Yichang 443002, Hubei, China. ${ }^{5}$ Affiliated Renhe Hospital of China Three Gorges University, Yichang 443001, Hubei, China.

Received: 20 June 2020 Accepted: 25 June 2021

Published online: 24 July 2021

\section{References}

1. Li Z, Jing $Y$, Li H, Xian $Y$, Wang $Y$. China's response to the rising stroke burden. BMJ. 2019:364:1879.

2. Wu SM, Wu B, Liu M, Chen ZM, Wang WZ, Anderson CS, et al. Stroke in China: advances and challenges in epidemiology, prevention, and management. Lancet Neurol. 2019;18(4):394-405. https://doi.org/10.1016/S14 74-4422(18)30500-3.

3. Khot SP, Morgenstern LB. Sleep and stroke. Stroke. 2019;50(6):1612-7. https://doi.org/10.1161/STROKEAHA.118.023553.

4. Battle DE. Diagnostic and statistical manual of mental disorders (DSM). Codas. 2013;25(2):191-2. https://doi.org/10.1590/s2317-17822013000200017.

5. Brown DL, Feskanich D, Sánchez BN, Rexrode KM, Schernhammer ES, Lisabeth LD. Rotating night shift work and the risk of ischemic stroke. Am J Epidemiol. 2009;169(11):1370-7. https://doi.org/10.1093/aje/kwp056.

6. Körner E, Flooh E, Reinhart B, Wolf R, Ott E, Krenn W, et al. Sleep alterations in ischemic stroke. Eur Neurol. 1986;25(Suppl 2):104-10. https://doi.org/10.11 59/000116090

7. Raven F, Van der Zee EA, Meerlo P. Havekes R. The role of sleep in regulating structural plasticity and synaptic strength: implications for 
memory and cognitive function. Sleep Med Rev. 2018;39:3-11. https://doi. org/10.1016/j.smrv.2017.05.002.

8. Mins KN, Kirsch D. Sleep and stroke. Sleep Med Clin. 2016;11(1):39-51. https://doi.org/10.1016/j.jsmc.2015.10.009.

9. Matthews EE, Arnedt JT, McCarthy MS, Cuddihy LJ, Aloia MS. Adherence to cognitive behavioral therapy for insomnia: a systematic review. Sleep Med Rev. 2013;17(6):453-64. https://doi.org/10.1016/j.smrv.2013.01.001.

10. Hohagen F, Rink K, Käppler C, Schramm E, Riemann D, Weyerer S, et al. Prevalence and treatment of insomnia in general practice. A longitudinal study. Eur Arch Psychiatry Clin Neurosci. 1993;242(6):329-36. https://doi. org/10.1007/BF02190245.

11. Shah SH, Engelhardt R, Ovbiagele B. Patterns of complementary and alternative medicine use among United States stroke survivors. J Neuro Sci. 2008;271(1-2):180-5. https://doi.org/10.1016/j.jns.2008.04.014.

12. Liu Y, Feng H, Liu W, Mao H, Mo Y, Yin Y, et al. Regulation action and nerve electrophysiology mechanism of acupuncture on arousal state in patients of primary insomnia. Zhongguo Zhen Jiu. 2017;37(1):19-23. https://doi.org/1 0.13703/j.0255-2930.2017.01.004.

13. Kim YS, Lee SH, Jung WS, Park SU, Moon SK, Ko CN, et al. Intradermal acupuncture on shen-men and nei-kuan acupoints in patients with insomnia after stroke. Am J Chin Med. 2004;32(5):771-8. https://doi.org/1 0.1142/S0192415X04002399.

14. Ding L, Wang J, Yang H, Yu J. "Governor vessel daoqi method of acupuncture" combined with estazolam for insomnia: a randomized controlled trial. Zhongguo Zhen Jiu. 2018;38(5):4633-7. https://doi.org/10.13 703/j.0255-2930.2018.05.003.

15. Lee SH, Lim SM. Acupuncture for insomnia after stroke: a systematic review and meta-analysis. BMC Complement Altern Med. 2016;16(1):228. https://doi. org/10.1186/s12906-016-1220-z.

16. Chen LC, Chen IC, Wang BR, Shao CH. Drug-use pattern of Chinese herbal medicines in insomnia: a 4-year survey in Taiwan. J Clin Pharm Ther. 2009;34(5):555-60. https://doi.org/10.1111/j.1365-2710.2 009.01038.x.

17. Frass M, Strassl RP, Friehs $H$, Müllner M, Kundi M, Kaye AD. Use and acceptance of complementary and alternative medicine among the general population and medical personnel: a systematic review. Ochsner J. 2012;12 (1):45-56.

18. Zhou $\mathrm{QH}$, Wang $\mathrm{HL}$, Zhou $X L$, Xu MB, Zhang HF, Huang LB, et al. Efficacy and safety of suanzaoren decoction for chronic insomnia disorder in adults: study protocol for randomised, double-blind, double-dummy, placebocontrolled trial. BMJ Open. 2017;7(4):e014280. https://doi.org/10.1136/ bmjopen-2016-014280.

19. Xie CL, Gu Y, Wang WW, Lu L, Fu DL, Liu AJ, et al. Efficacy and safety of Suanzaoren decoction for primary insomnia: a systematic review of randomized controlled trials. BMC Complement Altern Med. 2013;13(1):18. https://doi.org/10.1186/1472-6882-13-18.

20. Shang $K$, Zhang $X$. The clinical efficacy and PSQI comparison between Suanzaoren decoction therapy and acupuncture combined with Suanzaoren decoction therapy in the treatment of insomnia. Lishizhen Medicine and Materia Medica Research. 2010;21(8):2014-6. https://doi.org/10.3969/j.issn.1 008-0805.2010.08.077.

21. Zhang JX. Experimental study of acupuncture combined with Suanzaoren decoction in treating insomnia. Journal of Liaoning University of TCM. 2011; 13(5):221-2. https://doi.org/10.13194/j.junivtcm.2011.05.223.zhangjx.066.

22. Schulz KF, Altman DG, Moher D, CONSORT group. CONSORT 2010 statement: updated guidelines for reporting parallel group randomised trials. BMJ. 2010;340:c332. https://doi.org/10.1136/bmj.c332.

23. MacPherson H, Altman DG, Hammerschlag R, Li YP, Wu TX, White A, et al. Revised standards for reporting interventions in clinical trials of acupuncture (STRICTA): extending the CONSORT statement. Acupunct Med. 2010;28(2): 83-93. https://doi.org/10.1136/aim.2009.001370.

24. Neurology cerebrovascular disease study group of the Chinese Medical Association. Chinese guidelines for diagnosis and treatment of acute ischemic stroke 2018. Chin J Neurol. 2018;51(9):666-82.

25. Neurology cerebrovascular disease study group of the Chinese Medical Association. Chinese guidelines for diagnosis and treatment of acute intracerebral hemorrhage 2019. Chin J Neurol. 2019:52(12):994-1005

26. Kansagra S. Sleep disorders in adolescents. Pediatrics. 2020;145(Supplement 2):S204-9. https://doi.org/10.1542/peds.2019-2056 I.

27. Mollayeva T, Thurairajah P, Burton K, Mollayeva S, Shapiro CM, Colantonio A. The Pittsburgh sleep quality index as a screening tool for sleep dysfunction in clinical and non-clinical samples: a systematic review and meta-analysis. Sleep Med Rev. 2016;25:52-73. https://doi.org/10.1016/j.smrv.2015.01.009.

28. Baglioni C, Nissen C, Schweinoch A, Riemann D, Spiegelhalder K, Berger M, et al. Polysomnographic characteristics of sleep in stroke: a systematic review and meta-analysis. Plos One. 2016;11(3):e0148496. https://doi.org/1 0.1371/journal.pone.0148496.

29. Wong ML, Lau KNT, Espie CA, Luik Al, Kyle SD, Lau EYY. Psychometric properties of the sleep condition indicator and insomnia severity index in the evaluation of insomnia disorder. Sleep Med. 2017;33:76-81. https://doi. org/10.1016/.sleep.2016.05.019.

30. Ayis SA, Ayerbe L, Ashworth M. Evaluation of the hospital anxiety and depression scale (HADS) in screening stroke patients for symptoms: item response theory (IRT) analysis. J Affect Disord. 2018;228:33-40. https://doi. org/10.1016/j.jad.2017.11.037.

31. Kwah LK, Diong J. National Institutes of Health Stroke Scale (NIHSS). J Physiother. 2014;60(1):61. https://doi.org/10.1016/j.jphys.2013.12.012.

32. Li C, Mai Y, Dong M, Yin Y, Hua K, Fu S, et al. Multivariate pattern classification of primary insomnia using three types of functional connectivity features. Front Neurol. 2019;10:1037. https://doi.org/10.3389/ fneur.2019.01037.

33. Bagherzadeh-Azbari S, Khazaie H, Zarei M, Spiegelhalder K, Walter M, Leerssen J, et al. Neuroimaging insights into the link between depression and insomnia: a systematic review. J Affect Disord. 2019;258:133-43. https:// doi.org/10.1016/j.jad.2019.07.089.

34. Beloosesky Y, Grinblat J, Laudon M, Grosman B, Streifler JY, Zisapel N. Melatonin rhythms in stroke patients. Neurosci Lett. 2002;319(2):103-6. https://doi.org/10.1016/S0304-3940(01)02568-X.

35. Li TB. Effect of acupuncture on poststroke insomnia. Chin J Rehabil Theory Pract. 2007;13(7):656-7.

36. Streitberger K, Kleinhenz J. Introducing a placebo needle into acupuncture research. Lancet. 1998;352(9125):364-5. https://doi.org/10.1016/50140-6736 (97)10471-8.

37. Kleinhenz J, Streitberger K, Windeler J, Güssbacher A, Mavridis G, Martin E. Randomised clinical trial comparing the effects of acupuncture and a newly designed placebo needle in rotator cuff tendinitis. Pain. 1999;83(2):235-41. https://doi.org/10.1016/S0304-3959(99)00107-4.

38. Lu TY, Li Y, Pan JY, Wu DR. Study on minimal important difference of the Pittsburgh sleep quality index based on clinical trial of traditional Chinese medicine. Journal of Guangzhou University of Traditional Chinese Medicine. 2013;30(4):574-8. https://doi.org/10.13359/j.cnki.gzxbtcm.2013.04.020.

39. Maness DL, Khan M. Nonpharmacologic management of chronic insomnia. Am Fam Physician. 2015;92(12):1058-64.

40. Lang KM, Little TD. Principled missing data treatments. Prev Sci. 2018;19(3): 284-94. https://doi.org/10.1007/s11121-016-0644-5.

41. Dong YR, Peng CYJ. Principled missing data methods for researchers. Springerplus. 2013;2(1):222. https://doi.org/10.1186/2193-1801-2-222.

42. Leppävuori A, Pohjasvaara T, Vataja R, Kaste M, Erkinjuntti T. Insomnia in ischemic stroke patients. Cerebrovasc Dis. 2002;14(2):90-7. https://doi.org/1 $0.1159 / 000064737$

43. Palomäki H, Berg A, Meririnne E, Kaste M, Lönnqvist R, Lehtihalmes $M$, et al. Complaints of poststroke insomnia and its treatment with mianserin. Cerebrovasc Dis. 2003;15(1-2):56-62. https://doi.org/10.1159/ 000067127.

44. Duss SB, Seiler AS, Schmidt MH, Pace M, Adamantidis A, Müri RM, et al. The role of sleep in recovery following ischemic stroke: a review of human and animal data. Neurobio Sleep Circadian Rhythms. 2016;2:94-105.

45. Sterr A, Kuhn M, Nissen C, Ettine D, Funk S, Feige B, et al. Post-stroke insomnia in community-dwelling patients with chronic motor stroke: physiological evidence and implications for stroke care. Sci Rep. 2018;8(1): 8409. https://doi.org/10.1038/s41598-018-26630-y.

46. McDermott M, Brown DL, Chervin RD. Sleep disorders and the risk of stroke. Expert Rev Neurother. 2018;18(7):523-31. https://doi.org/10.1080/14737175.2 018.1489239.

47. Levin MF, Kleim JA, Wolf SL. What do motor "recovery" and "compensation" mean in patients following stroke? Neurorehabil Neural Repair. 2009;23(4): 313-9. https://doi.org/10.1177/1545968308328727.

48. Zhou QH, Zhou XL, Xu MB, Jin TY, Rong PQ, Zheng GQ, et al. Suanzaoren formulae for insomnia: updated clinical evidence and possible mechanisms. Front Pharmacol. 2018:9:76. https://doi.org/10.3389/fphar.2018.00076.

49. Gao B, Kilic E, Baumann CR, Hermann DM, Bassetti CL. Gammahydroxybutyrate accelerates functional recovery after focal cerebral 
ischemia. Cerebrovasc Dis. 2008;26(4):413-9. https://doi.org/10.1159/00015 683.

50. Gao J, Wang QM, Huang YW, Tang KL, Yang X, Cao ZW. In silico study of anti-insomnia mechanism for Suanzaoren prescription. Front Pharmacol. 2019;10:925. https://doi.org/10.3389/fphar.2019.00925.

51. Wei JJ, Yang WT, Yin SB, Wang C, Wang Y, Zheng GQ. The quality of reporting of randomized controlled trials of electroacupuncture for stroke. BMC Complement Altern Med. 2016;16(1):512. https://doi.org/10.1186/s12 906-016-1497-y.

52. Yin $X$, Dong $B$, Liang $T$, Yin $P$, Li X, Lin $X$, et al. Efficacy and safety of electroacupuncture on treating depression-related insomnia: a study protocol for a multicentre randomised controlled trial. BMJ Open. 2019;9(4): e021484. https://doi.org/10.1136/bmjopen-2018-021484.

53. Zhu W, Ye Y, Liu Y, Wang XR, Shi GX, Zhang S, et al. Mechanisms of acupuncture therapy for cerebral ischemia: an evidence-based review of clinical and animal studies on cerebral ischemia. J Neuroimmune Pharmacol. 2017:12(4):575-92. https://doi.org/10.1007/s11481-017-9747-4.

54. Xing Y, Zhang M, Li WB, Dong F, Zhang F. Mechanisms involved in the neuroprotection of electroacupuncture therapy for ischemic stroke. Front Neurosci. 2018;12:929. https://doi.org/10.3389/fnins.2018.00929.

55. Liu Q, Deng LL, Wang F, Wang LJ, Guo JH. Clinical research of high frequency electric acupuncture combined with resuscitation needling technique in the treatment of insomnia. Journal of Zhejiang Chinese Medical University. 2019;43(11):1284-7. https://doi.org/10.16466/j.issn.10055509.2019.11.020.

56. Xu L, Jiang LL, He F. Clinical study on treatment of chronic insomnia by electroacupuncture combined with low frequency repetitive transcranial magnetic stimulation. Jiangsu Journal of Traditional Chinese Medicine. 2021; 53(3):60-2. https://doi.org/10.19844/j.cnki.1672-397X.2021.03.022.

57. Lee SY, Baek YH, Park SU, Moon SK, Park JM, Kim YS, et al. Intradermal acupuncture on shen-men and nei-kuan acupoints improves insomnia in stroke patients by reducing the sympathetic nervous activity: a randomized clinical trial. Am J Chin Med. 2009;37(6):1013-21. https://doi.org/10.1142/S01 92415X09007624.

58. He W, Li M, Zuo L, Wang M, Jiang L, Shan H, et al. Acupuncture for treatment of insomnia: an overview of systematic reviews. Complement Ther Med. 2019:42:407-16. https://doi.org/10.1016/j.ctim.2018.12.020.

59. Zhu D, Bai L, Zhang $X, X u X$, Zhang J. Research progress on quantification of electroacupuncture parameters. Zhongguo Zhen Jiu. 2015;35(5):525-8. https://doi.org/10.13703/j.0225-2930.2015.05.032.

60. Kwon YB, Kang MS, Son SS, Kim JT, Lee YH, Han HJ, et al. Different frequencies of electroacupuncture modified the cellular activity of serotonergic neurons in brainstem. Am J Chin Med. 2000;28(3-4):435-41. https://doi.org/10.1142/S0192415X00000519.

61. Hou Z, Sun Z, Sun S. Impacts of the repetitive transcranial acupuncture stimulation on the content of serum orexin $A$ in patients with post-stroke insomnia. Zhongguo Zhen Jiu. 2018;38(10):1039-42. https://doi.org/10.13 703/j.0255-2930.2018.10.003.

62. Yi PL, Lu CY, Jou SB, Chang FC. Low-frequency electroacupuncture suppresses focal epilepsy and improves epilepsy-induced sleep disruptions. J Biomed Sci. 2015;22(1):49. https://doi.org/10.1186/s12929-015-0145-z.

63. Chen D, Guo H. Therapeutic effects of acupuncture combined with rehabilitation training on dysphagia in post-stroke pseudobulbar palsy. Zhongguo Zhen Jiu. 2018;38(4):364-8. https://doi.org/10.13703/j.0255-2930.2 018.04.006.

64. He W, Tong YY, Zhao YK, Li YW, Zhang L, Liu ZH. Analysis on current state of acupuncture clinical trials with sham acupuncture design in western countries. Zhen Ci Yan Jiu. 2015;40(5):423-6. https://doi.org/10.13702/j.10000607.2015.05.016.

65. Linde K, Niemann K, Schneider A, Meissner K. How large are the nonspecific effects of acupuncture? a meta-analysis of randomized controlled trials. BMC Med. 2010;8(1):75. https://doi.org/10.1186/1741-7015-8-75.

66. Powers WJ, Rabinstein AA, Ackerson T, Adeoye OM, Bambakidis NC, Becker $K$, et al. Guidelines for the early management of patients with acute ischemic stroke: 2019 update to the 2018 guidelines for the early management of acute ischemic stroke: a guideline for healthcare professionals from the American Heart Association/American Stroke Association. Stroke. 2019;50(12):e344-418. https://doi.org/10.1161/STR. 0000000000000211.

67. Liu F, Guo W, Liu L, Long Z, Ma C, Xue Z, et al. Abnormal amplitude lowfrequency oscillations in medication-naive, first-episode patients with major depressive disorder: a resting-state fMRI study. J Affect Disord. 2013;146(3): 401-6. https://doi.org/10.1016/j.jad.2012.10.001.

68. Huang $Z Y$, Liang $P P$, Jia $X Q$, Zhan $S Q$, Li N, Ding Y, et al. Abnormal amygdala connectivity in patients with primary insomnia: evidence from resting state fMRI. Eur J Radiol. 2012;81(6):1288-95. https://doi.org/10.1016/j. ejrad.2011.03.029.

69. Claustrat B, Brun J, Chazot G. The basic physiology and pathophysiology of melatonin. Sleep Med Rev. 2005;9(1):11-24. https://doi.org/10.1016/j.smrv.2 004.08.001.

70. Zhang W, Li F, Zhang T. Relationship of nocturnal concentrations of melatonin, gamma-aminobutyric acid and total antioxidants in peripheral blood with insomnia after stroke: study protocol for a prospective nonrandomized controlled trial. Neural Regen Res. 2017;12(8):1299-307. https:// doi.org/10.4103/1673-5374.213550.

71. Fiorina P, Lattuada G, Ponari O, Silvestrini C, DallAglio P. Impaired nocturnal melatonin excretion and changes of immunological status in ischaemic stroke patients. Lancet. 1996;347(9002):692-3. https://doi.org/10.1016/S01406736(96)91246-5.

72. Spence DW, Kayumov L, Chen A, Lowe A, Jain U, Katzman MA, et al. Acupuncture increases nocturnal melatonin secretion and reduces insomnia and anxiety: a preliminary report. J Neuropsychiatry Clin Neurosci. 2004;16 (1):19-28. https://doi.org/10.1176/jnp.16.1.19.

\section{Publisher's Note}

Springer Nature remains neutral with regard to jurisdictional claims in published maps and institutional affiliations.

Ready to submit your research? Choose BMC and benefit from:

- fast, convenient online submission

- thorough peer review by experienced researchers in your field

- rapid publication on acceptance

- support for research data, including large and complex data types

- gold Open Access which fosters wider collaboration and increased citations

- maximum visibility for your research: over $100 \mathrm{M}$ website views per year

At $\mathrm{BMC}$, research is always in progress.

Learn more biomedcentral.com/submissions 